\title{
Anomalous spreading behaviour of polyethyleneglycoldistearate monolayers at air/water interface
}

\author{
S JOHN COLLINS, ARUNA DHATHATHREYAN* and T RAMASAMI \\ Chemical Lab., Central Leather Research Institute, Adyar, Chennai 600020 , \\ India \\ e-mail: adhatha@indiaserver.com
}

MS received 11 September 2000; revised 13 November 2000

\begin{abstract}
Spreading behaviour of the dimeric surfactant polyethyleneglycoldistearate (PEGDS) monolayer at air/water interface has been studied using surface pressure-area $(\pi-A)$ isotherms as a function of temperature. The isotherms show a plateau suggesting a transition between a liquid expanded (LE) and a condensed state. The condensed state possibly arises due to nucleation and growth of multilayers from the monolayer. Isobaric measurements of both $A-T$ and $\pi-T$ at constant area show transitions at $T=295 \mathrm{~K}$. These plots suggest a melting followed by formation of condensed microcrystallites. Structure optimization carried out using various angles of orientation of the alkyl tails with respect to the backbone in PEGDS reveals tilt transitions of the tails in different states which can be related to the packing behaviour seen in the isotherms. Optical microscopy has been used to confirm the structures in these states.
\end{abstract}

Keywords. Anomalous behaviour; polythyleneglycoldistearate; air/water interface; surface pressure-area isotherms; condensed microcrystallites.

\section{Introduction}

When spread at the air/water interface, Langmuir monolayers of water-insoluble amphiphiles with simple polar groups can exist in different phases or states depending on the surface density (or surface pressure), temperature and surface structure. A number of techniques, including surface pressure $^{1}$ and surface potential ${ }^{2}$ measurements, fluorescence ${ }^{3-6}$. Brewster angle and electron microscopy ${ }^{7-11}$, X-ray diffraction and reflectivity ${ }^{12-14}$, neutron scattering/reflectivity ${ }^{15-17}$, external infrared reflection absorption spectrometry ${ }^{18}$ and atomic force microscopy of supported films ${ }^{19-21}$ have distinguished these monolayer states in terms of molecular ordering, including the positional (translational) order of the head groups and the orientational (tilt) order of the chains. This paper describes the spreading behaviour and the different states of polyethyleneglycoldistearate (PEGDS) monolayers at air/water interface.

PEGDS $\left(\mathrm{CH}_{3}\left(\mathrm{CH}_{2}\right)_{16} \mathrm{CO}\left(\mathrm{OCH}_{2} \mathrm{CH}_{2}\right) n \mathrm{O}_{2} \mathrm{C}\left(\mathrm{CH}_{2}\right)_{16} \mathrm{CH}_{3}\right) \quad(n=8)$ (scheme 1) can be considered as a dimeric $m-s-m$ surfactant (where $m$ and $s$ are the side alkyl chains and PEG the repeating units respectively).

Such dimeric surfactants can serve as good models for triblock amphiphilic copolymers ${ }^{22,23}$. Stearic acid being a good amphiphile enhances the HLB number of the

\footnotetext{
*For correspondence
} 


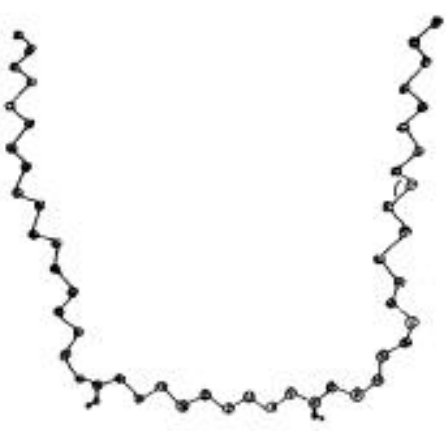

Scheme 1.

PEG-based polymer in these surfactants. Such PEG-based surfactants are attracting much interest in recent times because of their practical use and the potential they offer in industrial applications ${ }^{24}$. Solution properties of such end-capped low molecular weight PEG esters have been studied ${ }^{25-28}$.

In this work, experiments on PEGDS monolayers using surface pressure-molecular area $(\pi-A)$ isotherms and variations of $\pi$ and $A$ with temperature have been carried out to locate the boundaries between the states in surface pressure (or area/molecule) temperature space. Structure minimization has been carried out for various tilt angles of the alkyl chains with respect to the backbone of PEGDS to understand the conformational changes taking place during the phase transitions. Optical microscopy and TEM (selective area diffraction) have been used to study the morphology and packing of PEGDS in LB films.

\section{Experimental}

PEGDS was obtained from Sigma Chemicals, USA (99.9\% pure) and was used as such. Its molecular weight was 1000 . For the monolayer experiments PEGDS was dissolved in HPLC grade chloroform/methanol (1\% methanol) to obtain millimolar concentration. In all the experiments the subphase used was deionised water from a milliQ, millipore system. A commercial NIMA 611 trough fitted with a wilhelmy balance (accuracy $0.01 \mathrm{mN} / \mathrm{m}$ ) and a thermostat (accuracy $0.01^{\circ} \mathrm{C}$ ) was used for the isotherm measurements. All the isotherms were measured in the thermodynamic mode. In this mode, the barrier advances one step (1/1000 of the total compression) and waits until $\pi$ has equilibrated, that is until the digitized pressure value is twice the same within $0.5 \mathrm{~s}$. In order to ensure the film stability, varying amounts of the compound were spread and also different compression rates were used (in continuous compression mode). The surface potential-molecular area $(\Delta V-A)$ isotherm was measured using the vibrating plate method. The Langmuir-Blodgett films (LB films) of PEGDS were transferred onto quartz slides (for optical microscopy) and carbon-coated copper grids (mesh size 200) (for TEM studies) at $\pi=25 \mathrm{mN} / \mathrm{m}$ and the transfer was of X-type. The transfer rate for both upstroke and downstroke was $2 \mathrm{~mm} / \mathrm{min}$.

The transfer ratio was 0.95. Optical microscopy was carried out using a Jeneval polarizing microscope and the growth of crystallite zones and long range morphological features were studied. 
In this study, the structure optimization of the PEGDS molecules at air/water interface was carried out using the INSIGHT II-DISCOVER programme ${ }^{29}$. In this the molecule is first built with standard bond lengths, bond angles and dihedral angles and then charges and potentials are assigned to each atom or groups of atoms. The total potential energy of the molecule is the sum of the potentials due to stretching, bending, torsional, out of plane interaction, interaction due to bond-bond coupling, angle-angle coupling, bond angle coupling, angle-torsion coupling, out of plane coupling, Van der Waals, coulombic and hydrogen bonding interactions.

The potential energy of the molecule is given by,

$$
\begin{aligned}
E= & \Sigma_{b} D_{b}\left\{1-\mathrm{e}^{-a\left(b-b_{0}\right) 2}\right\}+1 / 2 \Sigma_{\theta} H_{\theta}\left(\theta-\theta_{0}\right)^{2}+\Sigma_{\phi}[1+s \cos (n \phi)]+1 / 2 \Sigma_{\chi} H_{\chi}{ }^{2} \\
& +\Sigma_{b} \Sigma_{b^{\prime}} F_{b b^{\prime}}\left(b-b_{0}\right)\left(b^{\prime}-b_{0}^{\prime}\right)+\Sigma_{\theta} \Sigma_{\theta}{ }^{\prime} F_{\theta \theta^{\prime}}\left(\theta-\theta_{0}\right)\left(\theta-\theta_{0}^{\prime}\right)+\Sigma_{b} \Sigma_{\theta} F_{b \theta}\left(b-b_{0}\right)\left(\theta-\theta_{0}\right) \\
& +\Sigma_{\varphi \theta \theta} \cos (\varphi)\left(\theta-\theta_{0}\right)\left(\theta-\theta_{0^{\prime}}\right)+\Sigma_{\chi} \Sigma_{\chi}{ }^{\prime} F_{\chi \chi^{\prime}} \chi \chi^{\prime}+\Sigma_{\varepsilon}\left[\left(r^{\prime} / r\right)^{1 / 2}-2\left(r^{\prime} / r\right)^{6}\right] \\
& +\Sigma_{i j} q_{i} q_{j} / \varepsilon r_{i j}+\Sigma_{i j}\left(C_{i j} / r_{i j}{ }^{2}-D_{i j} / r_{i j}{ }^{10}\right) .
\end{aligned}
$$

Here $D_{b}$ denotes dissociation energy, $\alpha$ the anharmonicity constant, $b o$ the equilibrium bond length, $H_{\theta}, H_{\varphi}$ and $H_{\chi}$ are the force constants. The first four terms in the above expression correspond to the energy of deformation of bond length, valence angles, torsional angles and out-of-plane interactions and are referred to as the diagonal terms of the valence force field. Conformation of the molecule was achieved by minimizing the starting structure. DISCOVER adjusts the velocities of the atoms to maintain a constant average kinetic energy using leapfrog integration algorithm ${ }^{30}$. The methodology used is as follows: The molecular system is a two dimensional array of PEGDS molecules at density near to close-packed regime under periodic boundary conditions (PBC). A similar array of water molecules are relaxed and associated as a bottom layer in contact with the polar groups. The whole structure is assigned a cell dimension which gives the packing density or the cross-sectional area per molecule. The molecular system is relaxed under PBC for minimum energy and run dynamics for the molecular conformation. Such methodology was also used by us to study phase transitions in octadecylmalonic acid monolayers ${ }^{31}$. Assigning pseudo atoms in united atom model reduces the running time.

The packing density of the molecules was reduced so that the average area per molecule is increased by $1 \mathrm{~A}^{2}$. The experimental procedure was repeated for different packing densities. The energy values and the final coordinates of the molecular systems after each run is stored for further processing to establish phase transition.

\section{Results and discussion}

Figure 1 shows the $\pi-A$ isotherms of PEGDS monolayers measured at different rates of compression but otherwise under identical conditions. For clarity only four of the measured curves with 3,10,100 and 300 min of sweeptime are shown, but they indicate clearly the trend with longer sweeptimes. The isotherm measured in the thermodynamic mode described above is exactly the same as with the isotherm measured with a sweep time of $100 \mathrm{~min}$ (given as solid line). It is seen that except for slight differences in the plateau pressure, the isotherms are quite identical. The figure also shows the surface potential-molecular area isotherm, with a phase transition clearly reflected in the isotherm. The surface potential increases rapidly from zero even in the region where the surface pressure is nearly zero and reaches a plateau before the plateau in the $\pi-A$ isotherm. 


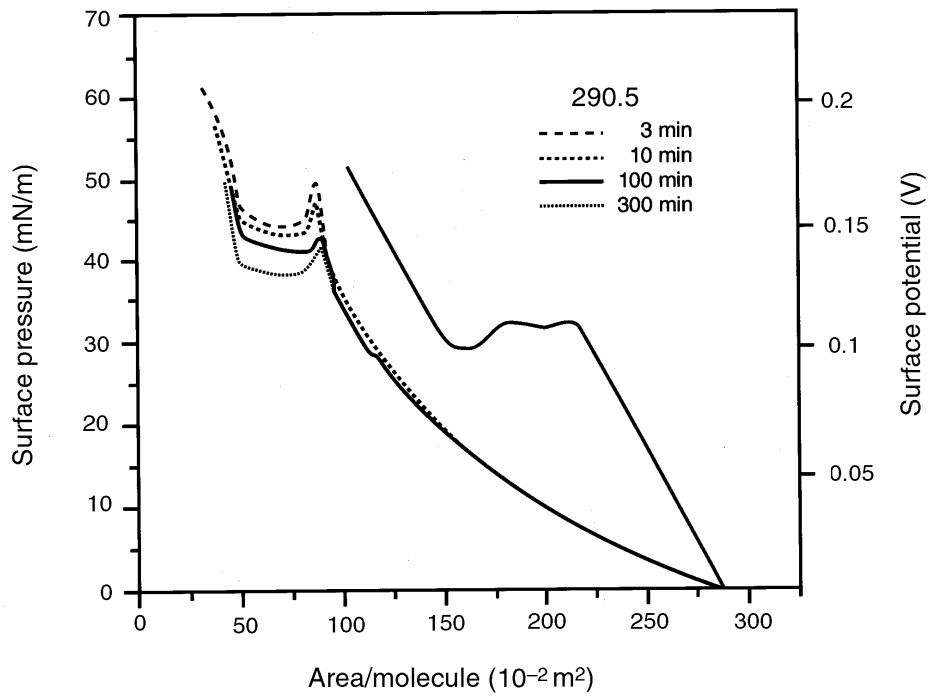

Figure 1. $\pi-A$ and $\Delta V-A$ isotherms of PEGDS on deionised water at room temperature.

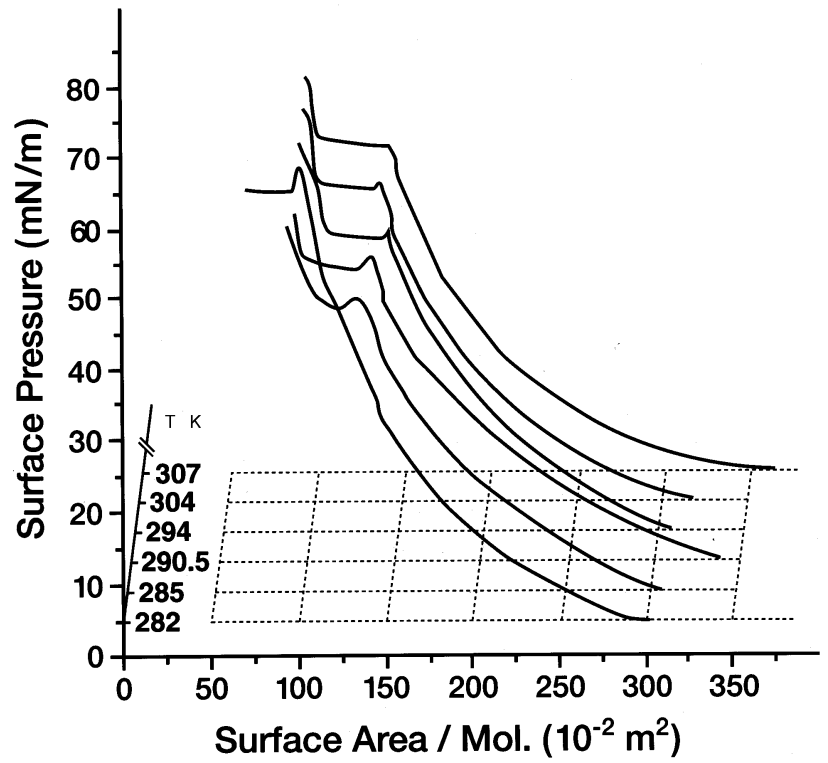

Figure 2. $\pi-A$ isotherm of PEGDS at air/water interface for different temperatures of the subphase (deionised water).

Figure 2 shows the $\pi-A$ isotherms of PEGDS monolayers at various temperatures measured with the thermodynamic mode. PEGDS forms quite stable monolayer and the general shape of the isotherm is the expanded type. 
The plateau seen here is generally not seen in oligomers ${ }^{32}$. De Gennes showed that in a $2 d$ melt, a single ideal chain could build up the $2 d$ bulk density without interpenetrating into other chains. In other words, $2 d$ segregated chains may exist ${ }^{33}$. Thus the plateau may be interpreted as arising due to a pressure induced reorganization and reorientation. Precise nature of these reorientations is of particular interest because they are the result of the balance between block-block (in the oligomer) and block-interface interactions. From isotherms a scheme for the general packing of the PEGDS molecules at air/water interface may be visualized. It is possible that in the gaseous state the tails and the backbone of PEGDS molecules lie near the interface. On compression the backbones take up a coiled structure with the cross sectional area gradually reducing while the stearyl tails are still near the interface. On further compression, the tails start moving away from the water surface and there is an increase in the available area for the backbone resulting in a small decrease in surface pressure. These results suggest that the backbone tends to be highly extended on water initially and will have a surface emergence 'footprint' of only one or two monomers in width. On compression these surface anchored groups are pushed closer and reorientation of the tails occur.

The larger areas with increase in temperature is indicative of an energy barrier that the molecules must overcome in order to leave the water surface and form a multilayer. This observed temperature dependence is consistent with decreased solubility seen for polyethyleneglycol with increase in temperature ${ }^{34,35}$.

Repeated compression and expansion cycles show hysteresis at the plateau and with continuous hysteresis cycle the hysteresis seems to reduce. This type of total respreading indicates the formation of microdomains. Reflection ellipsometric measurements ${ }^{36}$ with the ellipsometric phase shift $\delta \Delta$ were carried out on PEGDS for the different phases. However, $\delta \Delta$, a function of refractive index and film thickness is the sole angle that could be measured. Assuming the refractive index remains constant at a value of 1.5 for organic amphiphiles, values for thickness of the film at different molecular areas and surface pressure were estimated (table 1).

The assumption in the above estimates of thickness is that there is no strong inter or intramolecular interactions as the molecular area is reduced.

It has been found that the compressibilities of the materials in the condensed phase depend on the film balance they are usually measured with. To investigate this effect, several isotherms have been measured with different amount of material spread, but with initial area per molecule and compression rate per molecule kept constant. Table 2 shows the resulting compressibility for two different pressures.

An experiment wherin the surface pressure was maintained constant while molecular area was measured as a function of temperature $T$ was carried out and a plot of molecular

Table 1. Thickness of PEGDS monolayers.

\begin{tabular}{lcc}
\hline $\begin{array}{l}\text { Molecular area } \\
\left(10^{-20} \mathrm{~m}^{2}\right)\end{array}$ & $\begin{array}{c}\text { Surface pressure } \\
(\mathrm{mN} / \mathrm{m})\end{array}$ & $\begin{array}{c}\text { Film thickness } \\
(\AA)\end{array}$ \\
\hline 250 & 3 & $8 \cdot 5$ \\
175 & 14 & $12 \cdot 5$ \\
125 & 22 & $14 \cdot 5$ \\
110 & 35 & $17 \cdot 5$ \\
65 & 42 & $20 \cdot 9$ \\
\hline
\end{tabular}


Table 2. Compressibility of PEGDS monolayers.

\begin{tabular}{lcc}
\hline & \multicolumn{2}{c}{ Compressibility $(\mathrm{m} / \mathrm{mN})$ at pressure } \\
\cline { 2 - 3 } $\begin{array}{l}\text { Amount } \\
\text { spread }(\%)\end{array}$ & $30 \mathrm{mN} / \mathrm{m}$ & $45 \mathrm{mN} / \mathrm{m}$ \\
\hline 100 & $0 \cdot 243$ & $0 \cdot 430$ \\
80 & $0 \cdot 247$ & $0 \cdot 436$ \\
60 & $0 \cdot 247$ & $0 \cdot 440$ \\
40 & $0 \cdot 248$ & $0 \cdot 442$ \\
20 & $0 \cdot 250$ & $0 \cdot 450$ \\
\hline
\end{tabular}
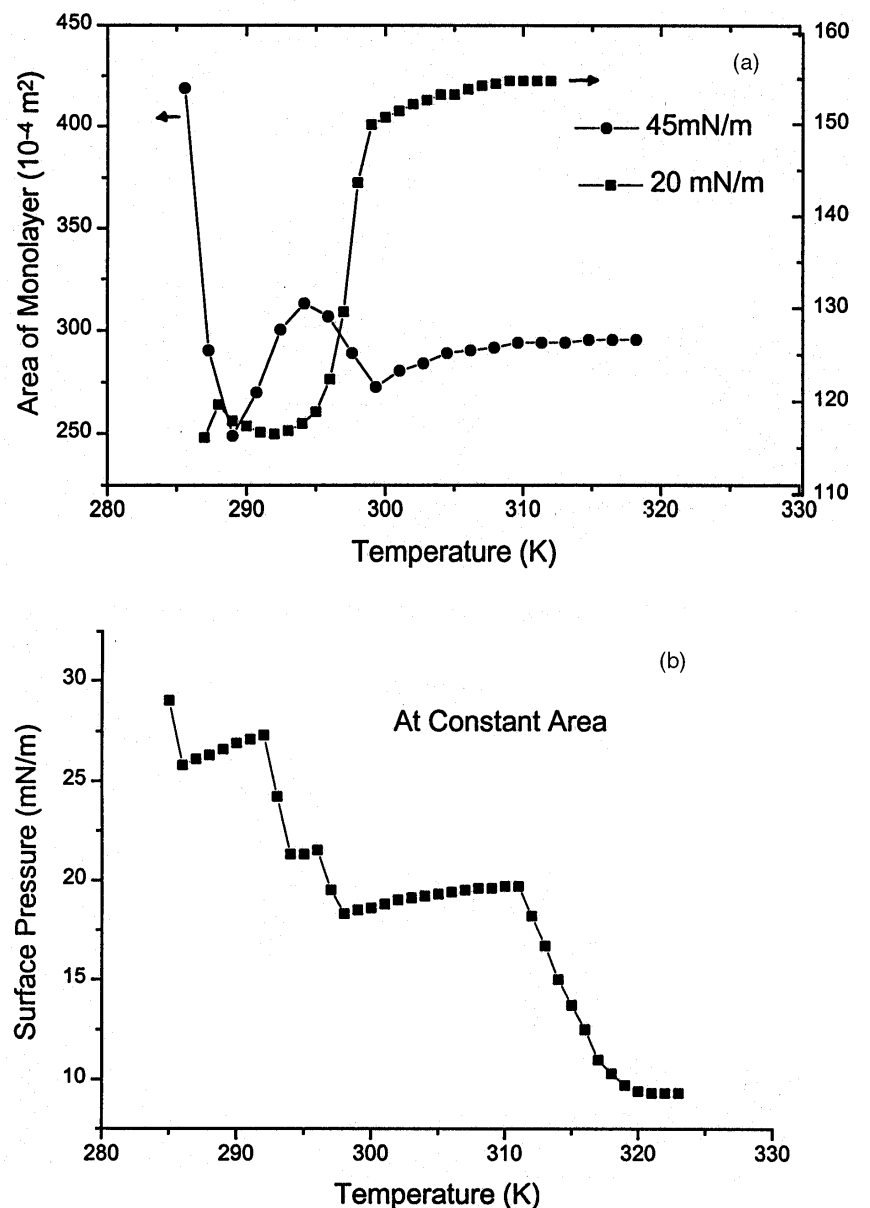

Figure 3. Plot of (a) $A-T$ (constant surface pressure), and (b) $\pi-T$ (constant area) for PEGDS.

area $A-T$ is shown in figure $3 \mathrm{a}$. Figure $3 \mathrm{~b}$ shows the $\pi$ versus the $T$ curve. In this experiment, by varying the temperature, the surface pressure was monitored keeping the molecular area constant. The plots suggest low temperature regions between 285 and 
$291 \mathrm{~K}$ and 298 and $311 \mathrm{~K}$ with a break at $295 \mathrm{~K}$. Since PEGDS has a melting transition at $313 \mathrm{~K}$, variable temperature experiments were carried out only till $311 \mathrm{~K}$.

\subsection{Structure minimization}

The minimized energy plotted by fixing various angles between the tails and the backbone of PEGDS is shown in figure 4. The plot shows three regions with transition at $120^{\circ}$ and $140^{\circ}$. This transition may be explained as follows: The PEGDS molecules have the alkyl chains bent at $140^{\circ}$ during the first phase of the compression. On further compression the backbones pack more closely. Since it requires more energy to tilt the alkyl chains beyond $140^{\circ}$, this angle remains stable while the molecular backbones coil around, thus reducing the area/molecule further.

\subsection{Optical microscopy}

In the $\pi-A$ isotherm, the expanded portion of the isotherm with a large area is followed by a long plateau corresponding to a region of $2 d$ aggregation. The monolayer appears heterogeneous on a macroscopic scale and interesting structures appear as are seen in the optical micrograph (figure 5a) of the PEGDS film immediately after transfer at the onset of the plateau. The domains exhibit striking patterns reminiscent of fractals or dendrites (figure 5a, bottom). This may be due to the instability arising from the slow diffusion of the molecules from a metastable state or a state with a lower stability to an equilibrium state under the given experimental conditions. This kind of exodiffusion is proportional to the concentration gradient. The ramified patterns are not very stable. The line tension that tends to minimize the perimeter of the interface between the 2 domains will eventually transform them into circles. This process does take place over many hours (figure 5b, top).

In conclusion, PEGDS shows a transition from an expanded to a condensed $2 d$ aggregates. The surface potential and the ellipsometric data confirm this transition. Both melting and the subsequent condensed microcrystalline domains are seen in the surface

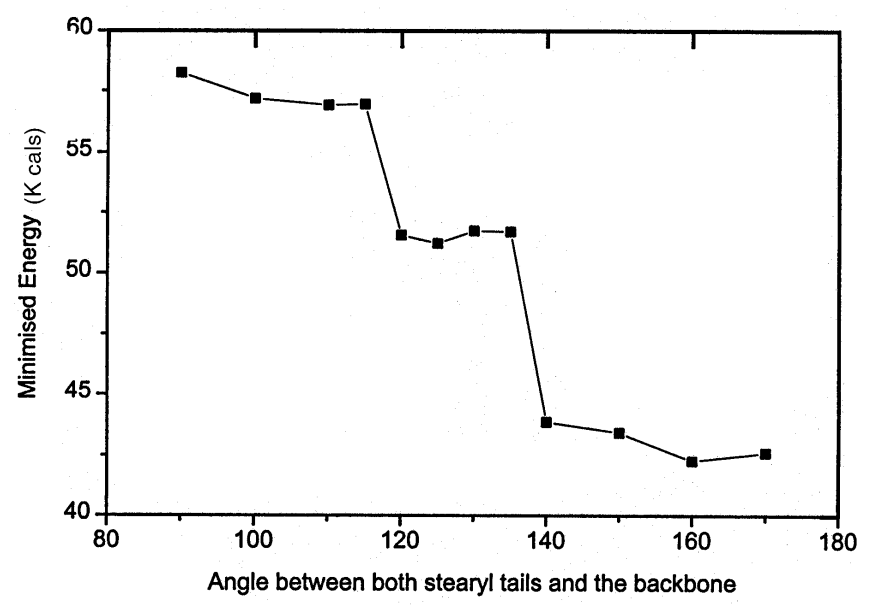

Figure 4. Plot of minimized energy for various tilt angles of the alkyl chains with respect to backbone of PEGDS at air/water interface. 

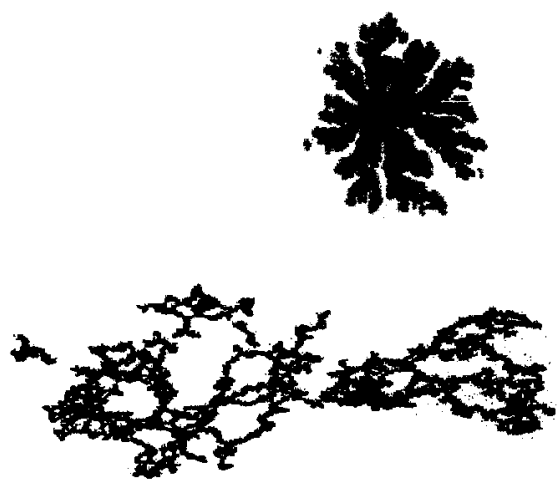

Figure 5. Optical micrograph of LB film of PEGDS (a) immediately after transfer and (b) after $6 \mathrm{~h}$.

pressure-temperature plots. Structure minimization carried out at various angles of orientation of the tails with respect to the backbone reveal the tilt transitions of the stearyl chains with respect to the backbone. These are further substantiated by optical microscopic studies.

\section{Acknowledgement}

The authors would like to thank the Department of Science and Technology, Government of India for a project grant under which this work was carried out.

\section{References}

1. Gaines G L Jr 1966 Insoluble monolayers at liquid-gas interfaces (New York, Wiley Interscience, )

2. Aveyard R and Haydon D A 1973 An introduction to the principles of surface chemistry (Cambridge: University Press)

3. McConnell H M, Tamm L K and Weis R M 1981 Proc. Natl. Acad. Sci. USA 1843249

4. Losche M and Mohwald H 1984 Rev. Sci. Instrum. 551968

5. Knobler C M 1990 Science 249870

6. Stine K J 1994 Micros. Res. Technol. 27439

7. Henon S and Meunier J 1991 J. Chem. Phys. 68936

8. Honig D and Mobius D 1991 J. Phys. Chem. 954590

9. Vollhardt D 1996 Adv. Colloid Interface Sci. 64143

10. Fischer A and Sackmann E 1985 Nature (London) 313299

11. Kajiyama T 1995 MRS Bull. 32

12. Pershan P R 1990 Faraday Discuss. 89231

13. Tippmann-Krayer P and Mohwald H 1991 Langmuir 72303

14. Shih M C, Bohanon T M, Mikrut J M, Zschack P and Dutta P 1992 Phys. Rev. A 455734

15. Als-Nielsen J, Jacquemain D, Kjaer K, Levellier F, Lahav M and Leiserowitz L 1994 Phys. Rep. 246252

16. Crowley T L, Lees E M, Simister E A, Thomas R K, Penfold J and Rennie A R 1990 Colloid Surf. 52252

17. Thomas R K and Penfold J 1996 Curr. Opin. Colloid Interface Sci. 123

18. Mendelsohn R M, Brauner J W and Gericke A 1995 Annu. Rev. Phys. Chem. 46305

19. Hansma H G, Gould S A C, Hansma P K, Gaub H E, Longo M L and Zasadzinski J A N 1991 Langmuir 71051 
20. Schaper A E, Wolthaus L, Mobius D and Jovin T M 1993 Langmuir 92178

21. Meine K, Vollhardt D and Weidemann G 1998 Langmuir 141815

22. Menger F M and Littau C A 1995 J. Am. Chem. Soc. 11510083

23. Alami E, Beinest G, Marie P and Zana R 1993 Langmuir 91468

24. Anderson B and Olofson G 1987 Colloid Poly. Sci. 265318

25. Danino R, Talmon Y and Zana R 1995 Langmuir 111448

26. Zana R and Talmon Y 1993 Nature (London) 362228

27. Padmavathi N Ch and Chatterjee P R 1995 Langmuir 11767

28. Pollard M L, Pan R, Steiner C and Maldarelli C 1998 Langmuir 147222

29. DISCOVER 2.5 Manual, 2-23-90 Biosym Inc

30. Toxevaerd S 1991 Mol. Phys. 72159

31. John Collins S, Dhathathreyan A and Ramasami T 1998 J. Colloid Interface Sci. 203248

32. Nakamae K, Takeya T, Fujimara Y, Sakai I and Matsumoto T 1982 J. Macromol. Sci. B21 157

33. de Gennes P G 1979 Scaling concepts in polymer physics, Cornell University Press, London p 60-61

34. Boury E, Guilk A, Dedieu J and Proust J E 1994 Langmuir 101654

35. Minones J, Irirbarnegarey E, Varela C, Vila N, Conde O, Cid L and Cassas M 1992 Langmuir 82781

36. Karlstrom G and Engkrist O 1997 In Polyethyleneglycol: Chemistry and biological application (eds Harris J M and Zalipsky S) Am. Chem. Soc., Washington DC, Ch 2 